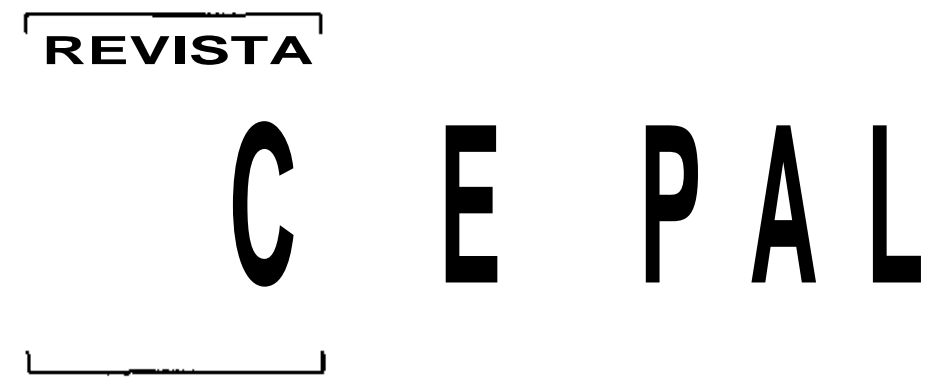

NUMERO 65

AGOSTO 1998

SANTIAGO DE CHILE

ÓSCAR ALTIMIR

Director

EUGENIO LAHERA

Secretario Técnico

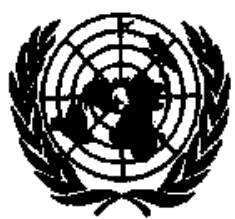

NACIONES UNIDAS 
Distribución del ingreso, pobreza y gasto social en América Latina José Antonio Ocampo

Gasto militar y el desarrollo en América Latina

Eugenio Lahera y Marcelo Ortúzar

Crecimiento, justicia distributiva y política social

Andrés Solimano

Equidad, inversión extranjera y competitividad internacional

Adolfo Figueroa

Tensiones en el ajuste estructural en América Latina: asignación vs. distribución

Daniel M. Schydlowsky

Competitividad y regulaciones laborales

Luis Beccaria y Pedro Galín

Familias latinoamericanas: convergencias y divergencias de modelos y políticas

Irma Amagada

Los acuerdos de libre comercio y el trabajo de las mujeres:

el caso de Chile

Alicia Frohmann y Pilar Romaguera

Evolución macroeconómica del Paraguay 1989-1997:

burbuja de consumo y crisis financiera

119 L.-

Siéphane Straub

Estrategias de las empresas mexicanas en sus procesos de internacionalización

Alejandra Salas-Porras

La regulación de la prestación privada de servicios de agua potable y alcantarillado

Terence R. Lee y Andrei S. Jouravlev

Promoción de la calidad para mejorar la competitividad $169 v^{\prime}$ Hessel Schuurman 


\begin{tabular}{c|c|c|}
\cline { 2 - 3 } & REVISTA DE LA CEPAL 65 \\
\hline
\end{tabular}

\section{Equidad, inversión extranjera y competitividad internacional}

\section{Adolfo Figueroa}

Departamento de Economía,

Pontificia Universidad

Católica del Perú,

Lima
¿Es el grado de competitividad de los países independiente de su grado de desigualdad? ¿Es la competitividad una cuestión sólo de eficiencia microeconómica y sectorial, del tipo de cambio real, o es también una cuestión social? Hasta ahora los estudios especializados han hecho abstracción del problema de la equidad en la determinación de la competitividad de los países. Pero entonces no han podido explicar plenamente la competitividad observada. En este artículo se incorpora la equidad en la función de producción y también en las decisiones de los inversionistas en un mundo de perfecta movilidad del capital. Las predicciones del sistema teórico propuesto son, en general, consistentes con los datos observados en la economía mundial. En particular, América Latina presenta el mayor grado de desigualdạd entre las regiones del mundo; su participación en los flujos de la inversión extranjera directa es baja, y también lo es su participación en el comercio mundial (su competitividad). La teoría que se presenta aquí y los datos recogidos sugieren que las productividades relativas de los países dependen positivamente de la asignación de las inversiones y que esta asignación a su vez depende, también positivamente, de los grados de equidad en ellos. Luego, la competitividad de un país depende, entre otros factores, de su grado de desigualdad económica. Las sociedades compiten en el mercado de capitales, buscando atraer las inversiones privadas para así hacerse competitivas en el mercado de bienes; en esto, entre otros factores, influye su grado de equidad actual. 
I

\section{Introducción}

La competitividad internacional es uno de los temas favoritos en la literatura económica reciente. Como dice Krugman (1995), con este tema hay una obsesión que le parece peligrosa. Se piensa que hay una relación entre las diferencias de productividad — base de la competitividad - y el nivel de vida de los países, relación que Krugman encuentra inaceptable, tanto desde el punto de vista lógico como del empírico: el nivel de vida de un país dependería de su propia productividad (de su productividad absoluta), y no de su productividad relativa.

¿Qué se puede decir en cuanto a la relación entre competitividad y equidad? ¿Será acaso que la equidad también depende sólo de la productividad absoluta y es entonces independiente de las productividades relativas, es decir, de la competitividad? ¿O será al revés: que la competitividad depende del grado de equidad que la sociedad tiene? Hay abundante literatura económica que examina la equidad y la competitividad por separado, pero son muy pocos los trabajos sobre las interrelaciones de ellas. Este artículo intenta avanzar en este terreno.

La productividad de un país es un factor importante de la competitividad en los mercados internacionales. Pero, ¿cuál es la relación entre productividad y equidad? Para responder a esta pregunta, que es central en el presente estudio, se desarrolla aquí una teoría de la producción en la cual la equidad es una variable de la función de producción (sección II); se examina la lógica de los inversionistas frente al riesgo-país (sección III); se ponen a prueba empírica las predicciones del sistema teórico (sección IV); se analiza el papel de la dotación de recursos naturales (sección $\mathrm{V}$ ); y, por último, se presentan algunas conclusiones y se reseñan las perspectivas que abre la relación equidad-competitividad (sección VI).

\section{II}

\section{Equidad y productividad}

La competitividad de un país se puede definir como su capacidad de ganar posiciones en los distintos mercados internacionales. La teoría es que, en el largo plazo, esa capacidad depende de la productividad relativa del país. ¿Y cuáles son los factores que determinan la productividad de un país? Se puede sugerir aquí un conjunto de hipótesis teóricas al respecto.

Primero, la productividad dependería de todo el sistema productivo. Como se podría derivar del sistema tecnológico de Leontief, la productividad de un sector no puede ser independiente de la productividad

L. Este estudio fue iniciado en la Oficina Regional de la Organización Internacional del Trabajo (OIT) en Lima, hacia fines de 1995, gracias a una invitación de Víctor Tokman. Varios colegas han comentado sus sucesivas versiones. Quiero agradecer a David Drukker, Javier Iguiñiz, Ricardo Infante, Félix Jiménez, José Oscálegui y John Shehan, y de manera muy especial a Osear Altimir. También deseo agradecer al panel de comentaristas de la Latin American Studies Association (LASA) -Guadalajara, 17 a 19 de abril de 1997—, donde presenté este estudio por primera vez. La responsabilidad por los errores es, naturalmente, sólo mía. de los demás sectores. La infraestructura vial, los servicios de transporte, los puertos, los servicios de comunicaciones son factores que también influyen en la productividad del sistema productivo. Esto ha sido reconocido en varios estudios efectuados en América Latina. Así, los trabajos de la CEPAL sobre la transformación productiva con equidad (CEPAL, 1990) consideran que la productividad es una cuestión sistémica. De hecho, si se considera que en el sistema productivo hay interrelaciones de los sectores — como las del sistema tecnológico de Leontief- esa conclusión es inobjetable. Incluso el concepto de "intensidad de los factores" tendría que ser definido en relación con el contenido total — directo e indirecto- de capital y trabajo por unidad de producto, y no en relación sólo con los coeficientes directos, como se hace usualmente.

Segundo, la productividad de la economía dependería no sólo de la intensidad de los factores de producción, sino también de los cambios en la calidad de esos factores y en el conocimiento tecnológico. 
Tercero, la productividad también dependería de la calidad de los empresarios. Este es tal vez el factor limitante más significativo. Se necesitan "empresarios walrasianos," es decir, aquellos que revisan continuamente sus métodos de producción y adoptan las novedades tecnológicas que se vienen generando fuera de la firma, así como los nuevos productos. Pero se necesitan también, y de manera prioritaria, "empresarios schumpeterianos," que son los que desarrollan nuevos métodos de producción, con nuevas prácticas e insumos, nuevos productos, nuevos mercados, nuevas fuentes de insumos. Y como el marco institucional en el que operan las firmas sería igualmente importante para la eficiencia, se necesitarían empresarios schumpeterianos también en el sector público. Ellos tendrían que generar innovaciones institucionales que permitieran al sistema productivo elevar su nivel de productividad.

Los factores mencionados hasta aquí han sido examinados en la literatura económica. En este estudio se introducirá una nueva proposición teórica: la productividad dependería de la inversión, y ésta de la estabilidad social y política, la cual dependería a su ve?. del grado de equidad alcanzado por la sociedad. El desafío consiste pues en introducir la equidad en la función de producción y poder luego falsear empíricamente esta teoría.

Postularemos, para iniciar el argumento, una función de producción de la forma siguiente:

$$
Q_{t}=f\left(L_{t} ; S_{t-1}, Z_{t-1}\right)
$$

donde $Q$; es la cantidad producida de un bien en el periodo $t, L$ es la cantidad de trabajadores utilizados en el mismo período, $\mathrm{S}$ es un vector que representa el acervo de factores productivos privados - tierra, capital físico y capital humano (mano de obra con distintos grados de calificación) - y Z es un vector que representa el acervo de factores productivos públicos, ambos tipos de acervos medidos al final del período anterior.

Introducimos así los "bienes públicos" en la función de producción. Este conjunto de bienes incluye no sólo infraestructura y conocimiento tecnológico de dominio público (es decir, apropiable), sino también orden social. Se supone que el orden social es un bien público: una vez que está presente, nadie puede ser excluido de sus beneficios. El orden social entra a la función de producción porque sin orden social el proceso productivo no podría repetir, período tras período, la misma cantidad de producto utilizando la misma cantidad de insumos.
Los acervos señalados se acumulan mediante la inversión privada y la inversión pública. Supondremos que tales inversiones demoran un período en acumular los acervos, por lo cual éstos aparecen con un retraso de un período en la función de producción. También supondremos que las inversiones privadas y públicas incorporan innovaciones tecnológicas y dan lugar a la acumulación tanto de viejos como de nuevos bienes productivos. Por lo tanto, no es posible agregar vectores de períodos distintos en un número (y llamar al resultado "cantidad de capital"), pues ellos incluyen acervos de bienes productivos (capital físico y humano) que son heterogéneos y de calidades cambiantes.

Dadas las dificultades lógicas que plantea el problema de agregación del capital en una economía dinámica, la función de producción podría expresarse sólo en términos de la relación entre el producto y la cantidad de trabajadores, relación que cambia continuamente con la inversión. Esta es la formulación que utilizaremos aquí.

Si aceptamos que el orden social ingresa a la función de producción, ¿cuáles son los factores que determinan el orden social? En otro estudio (Figueroa, 1993) he desarrollado la teoría de que el orden social depende fundamentalmente del grado de equidad en la distribución de la riqueza. La proposición axiomática es que no toda distribución de la riqueza es socialmente tolerada. Sólo un subconjunto estricto de las posibles distribuciones de la riqueza sería aceptado socialmente; si el funcionamiento de la economía llevara a una solución fuera de esos límites, la sociedad entraría en una crisis distributiva, es decir, en el desorden social.

La crisis distributiva depende no sólo de los resultados distributivos del mercado, sino de la política redistributiva del Estado. El gasto público social sería un mecanismo del que dispone el Estado para reducir las brechas distributivas originadas en el funcionamiento del mercado y asegurar así el orden social.

Una creciente pauperización de las masas (sea absoluta o relativa) que condujera a la sociedad a una situación de crisis distributiva generaría nuevas formas de redistribución, en las cuales el derecho de propiedad ya no se respetaría plenamente. En tal situación, los ingresos no contractuales adquirirían importancia creciente. Como consecuencia, los niveles de violencia, corrupción e incertidumbre en el sistema productivo se elevarían. Todo ello significaría costos económicos para la sociedad. En el corto plazo, se acrecentarían los costos de la seguridad personal y de la protección de la propiedad, así como los costos de las paralizaciones por huelgas y todo tipo de desorden social. Estos costos se trasladarían a los costos de 
producción de las firmas. Parte de los costos sociales se transformarían entonces en mayores costos unitarios de éstas, con pérdida de competitividad.

En el largo plazo, los costos económicos de una crisis distributiva incluirían una retracción de la inversión privada (a la cual nos referiremos más adelante) y también una baja en la calidad de la mano de obra. Con la pauperización de las masas, por ejemplo, la desnutrición infantil aumenta. Se sabe que la capacidad de aprendizaje de las personas se determina en gran medida en la primera infancia, de modo que la calidad de la mano de obra futura se determina hoy. Por lo tanto, será mucho más costoso elevar la productividad del trabajador que haya tenido una infancia descuidada. La tasa de repitencia escolar y la morbilidad' serían mayores; por ende, la inversión en educación y salud necesaria para obtener un tipo particular de mano de obra sería también mayor.

En suma, nuestra proposición teórica es que la productividad depende, entre otros factores, de la estabilidad de la sociedad. Este último factor depende a su vez del grado de equidad económica que exista en la sociedad: si el grado de equidad disminuye, mayor será el riesgo de caer en la inestabilidad social y política, y menor productividad mostrará el sistema productivo. La equidad $(£)$ es un elemento de la función de producción de los distintos bienes.

En el corto, plazo, con acervos dados de bienes privados y públicos y un nivel dado de conocimientos tecnológicos, la función de producción se puede escribir así:

$$
Q,=n F\left(L_{t}\right)
$$

donde $n \approx n(E), y$ donde

$$
\begin{aligned}
& n=1 \text { si } E \geq E^{*} \\
& 0<n<I \text { si } E<E^{*}
\end{aligned}
$$

Hay crisis distributiva cuando la equidad $(E)$ toma un valor por debajo del umbral de tolerancia social a la desigualdad (£*). En este caso, las empresas tendrían que utilizar recursos para proteger la propiedad privada y también para reducir los costos de transacciones (entendidos como los mayores riesgos asociados al intercambio laboral, es decir, la mayor desconfianza en las relaciones laborales) que se habrían elevado. En una situación de crisis distributiva, la productividad laboral caería. La curva que describe la función $F$ de la ecuación (2) se desplazaría hacia abajo. ${ }^{1}$

En el largo plazo, la función de producción se puede expresar así:

$$
\begin{aligned}
& Q,=m, F\left(L_{i}\right. \\
& \text { donde } m>i .
\end{aligned}
$$

Suponemos que la función $F$ sólo puede desplazarse hacia arriba, de acuerdo al valor que tenga la variable $n i$. La productividad laboral dependería de los valores que adopte la variable $m$.

¿Qué factores determinan m? Proponemos la hipótesis de que las variables que mueven la función $F$ son la inversión privada (/) y la inversión pública $(\mathrm{G})$, pues es a través de estos gastos que se expande el acervo de factores productivos, tanto privados como públicos. Supondremos que el nuevo conocimiento tecnológico, obtenido a través de la inversión, está incorporado tanto en el capital físico como en el capital humano. Entonces, se tiene:

$$
{ }^{m},=\phi\left(I_{t-1}, G_{t-1}\right)
$$

La inversión pública la supondremos exógenamente determinada, pero la inversión privada será considerada endógena. De las relaciones presentadas hasta aquí se desprende que en el largo plazo la productividad laboral depende de las inversiones, pues éstas desplazan la función $F$ hacia arriba de manera continua.

\section{III}

\section{La lógica de los inversionistas}

Pero, ¿cuáles son tos factores que determinan las inversiones privadas de largo plazo en un país? Necesitamos una teoría de la inversión.

Sobre la inversión privada de largo plazo hay hasta tres teorías: la primera diría que la inversión es endógena al proceso económico; la segunda, que es endóge-

\footnotetext{
1 Sustituyendo equidad por salario real se puede expresar de manera más analítica lo que se ha dado en llamar la teoría de los "salarios de eficiencia" (Solow, 1990). Si los salarios reales cayeran por debajo de un umbral, la productividad laboral caería. Según esta teoría, la productividad laboral dependería del nivel del salario real (y no al revés como sostiene la teoría microcconómica convencional).
} 
na al proceso económico y también al sociopolítico; la tercera, que es totalmente exógena. En el primer caso la teoría establece que la inversión privada depende de la tasa de interés y de los rendimientos esperados, los que dependen sólo de precios relativos esperados (Barro, 1990). En el segundo se introduce el supuesto de que los rendimientos esperados dependen también del grado de estabilidad del sistema sociopolítico (Alesina y Perotti, 1993; Figueroa, 1993). En el último caso, los inversionistas sólo se guiarían por sus "animal spirits", como señaló Keynes.

Aquí adoptaremos la segunda teoría. Asumiremos que la decisión de inversión depende no sólo del proceso económico sino también del proceso sociopolítico de la sociedad, es decir, del orden social. Adoptar la tercera teoría significaría suponer que el crecimiento económico depende del estado de ánimo de los capitalistas, ante lo cual no habría nada que hacer en materia de política económica.

Diremos entonces que ante los riesgos que enfrenta un inversionista, su decisión de invertir dependería de la rentabilidad esperada y de su capacidad para absorber riesgos. Cuanto mayor sea esta capacidad, más entrará el inversionista a juegos con mayor retorno esperado, aunque sean más riesgosos. Supondremos que esta capacidad está limitada por la cantidad de activos con que cuenta el inversionista; es decir, que la aversión al riesgo es parte de sus restricciones y no de sus preferencias. Así los inversionistas con más activos tomarían mayores riesgos (Figueroa, 1993).

Supondremos también que el capital es un factor móvil entre países. Entonces, ¿cómo deciden los inversionistas a qué países asignan sus fondos? Para responder a esta pregunta se presenta aquí un modelo sobresimplificado. Consideraremos dos tipos de riesgos, el riesgo-producto y el riesgo-país, pues el inversionista debe decidir en qué producto y en qué país ha de invertir. En ambos casos el inversionista enfrenta dos posibles situaciones. En el caso del producto, puede obtener un buen retorno ( $r^{\wedge} \mathrm{o}$ un mal retorno $\left(\mathrm{r}_{2}\right)$. Luego, el retorno esperado de su inversión sería

$$
i^{*}=P_{i} r_{i}+P_{2} r_{2}, P_{i}+P_{2}=1, r,>r_{2}>0
$$

donde $P_{i}$ y $P_{2}$ se pueden interpretar como las probabilidades de que se produzca una u otra de estas situaciones.

Supondremos que los factores productivos privados y públicos son complementarios. Por lo tanto, para valores dados de las probabilidades, los retornos esperados dependerán de la inversión pública $(\mathrm{G})$.

En la decisión sobre el país, también supondremos dos situaciones: en el país $j$ se puede tener estabilidad sociopolítica, o una situación de inestabilidad y caos. Digamos que las probabilidades son $V_{i}$ y $V_{2}$ respectivamente, donde $V_{i}+V_{2}=1$. Supongamos que si se da la primera situación, el inversionista obtiene $r^{e}$, pero si se da la segunda su retorno es cero. Luego, su retorno esperado, tomando en cuenta el efecto de riesgo-país, sería

$$
R_{j}^{e}=V_{l j}{ }^{e}
$$

En términos del retorno esperado, el inversionista invertiría en el país con el mayor valor de $R^{e}$. es decir, en el país con la mayor estabilidad sociopolítica.

En el modelo anterior, la tasa de retorno esperado para el producto era independiente del país. Si se relaja este supuesto, el retorno en el país $j$ sería:

$$
R_{j}^{e}=V_{l i} r_{j}
$$

Todavía el inversionista invertiría en el país que ofreciese el más alto valor de $R^{e}$, pero ahora podría ocurrir que un país con relativa inestabilidad (un bajo valor de $V_{i}$ ) pudiera atraer inversiones debido a que el valor de $r$ " es relativamente alto.

En ambos modelos el riesgo es el mismo: perder toda la inversión si ocurre una inestabilidad sociopolítica. Es decir, el riesgo lo pone el factor de riesgo-país. $\mathrm{Si}$ el inversionista tuviera la capacidad de absorber esa eventual pérdida, él haría su inversión en el país con el más alto valor de $R^{e}$. Si no tuviera esa capacidad, no invertiría en país alguno.

$\mathrm{Si}$ se introduce en este sistema de relaciones la proposición teórica de que la probabilidad de tener estabilidad sociopolítica — es decir, orden social— depende del grado de equidad, se tiene que el retorno de la inversión $R^{e}$ no sería independiente del grado de equidad de la sociedad. Luego, la inversión privada, además de depender de la inversión pública, dependerá también del grado de equidad de la sociedad $(\mathfrak{f})$; es decir, la función de inversión tomaría la forma siguiente:

$$
\begin{aligned}
& 1=H(G) \quad \text { sí } E>E^{*} \\
& =J \quad(G, E) \quad \text { si } E<E^{*}
\end{aligned}
$$


donde $E^{*}$ es el umbral de la equidad socialmente tolerada.

La inversión privada no podría ser independiente del grado de equidad existente en la sociedad. Si el grado de equidad estuviera por encima del umbral de la tolerancia social, la inversión no se vería afectada por los cambios en la equidad; pero si el grado de equidad cayera por debajo de ese umbral, o estuviera en riesgo de caer, la inversión disminuiría. Es suficiente suponer que los inversionistas tienen distinta capacidad para absorber pérdidas — distintos "puntos de desastre", diría Hicks (1989) — para generar una curva donde hasta $E^{*}$ la relación entre inversión y equidad es positiva. Cuanto menor sea la equidad (y la estabilidad social), mayor riesgo corre el retorno de la inversión; luego, sólo invertirían grandes inversores, aquellos con capacidad para absorber las eventuales pérdidas. A medida que aumente $E$, el riesgo disminuiría e ingresarían inversores con menor capacidad para absorber pérdidas. Es claro que más allá de $E^{*}$ la curva se vuelve plana. ${ }^{2}$

Luego, en una sociedad muy desigual, el sistema de ecuaciones (3), (4) y (8) lleva a una función de producción que tendría la forma siguiente:

$$
Q_{t}=f\left(L_{t}, G_{t \cdot 1}, E_{t-1}\right) \text {, donde } E<E^{*}
$$

El nivel de la producción depende de la cantidad de trabajadores empleados en el mismo período, y también de la inversión pública y del nivel de equidad (cuando éste es inferior al umbral de tolerancia social), ambas variables del período anterior. Así, diremos que un proceso productivo puede ser más o menos "intensivo en equidad" comparado con otro, según sea el grado de estabilidad social que admite la inversión privada. Esto último depende de cuanto eslabonamiento tiene este proceso productivo con los demás sectores de la economía. Cuanto más eslabonamientos tenga, mayor será su necesidad de estabilidad social y, por lo tanto, será más intensivo en equidad.

Los inversionistas se dirigirían a explotar las ventajas absolutas, las ventajas comparativas o las venta- jas competitivas del país elegido. ${ }^{3}$ Su lógica al decidir estaría guiada por el modelo desarrollado aquí. Pero sus inversiones contribuirían a desarrollar esas ventajas para el futuro, lo que generaría un efecto dinámico. Cuando el proceso productivo de un bien es menos intensivo en estabilidad social, la inversión puede ser menos sensible al riesgo-país y en tal caso podría dirigirse a producir ese bien en enclaves (minas, petróleo, manufactura de maquila, centros de turismo). Pero si el proceso productivo en cuestión es intensivo en estabilidad social, el riesgo-país puede tener un efecto muy importante, por lo cual no se invertiría en el bien respectivo. Debido al factor de riesgo-país, al cual subyace el grado de equidad, las ventajas competitivas de un país podrían no desarrollarse y las ventajas comparativas no evolucionar.

En esta formulación teórica, la equidad resulta del funcionamiento del mercado en el período anterior (que opera, naturalmente, con variables exógenas) y de las políticas sociales del gobierno, que es también una variable exógena. Pero la teoría que quiero proponer aquí es que en una sociedad cuyo proceso de desarrollo tiene como condición inicial una marcada desigualdad, ésta se mantendrá. El mercado no podrá reducirla, el sistema político no tiene incentivos para hacerlo y la situación de desigualdad tiende a persistir. Se da así en esta sociedad un estado de desigualdad pronunciada.

De este sistema teórico se puede derivar una predicción con contenido empírico: sociedades con un alto grado de desigualdad recibirán poca inversión extranjera directa; a igualdad de condiciones, un país con mayor equidad atraería una mayor proporción de la inversión privada, con lo cual elevaría la productividad y mejoraría su participación en el mercado internacional. Otra predicción empírica que se deriva de esta teoría es que en economías muy inestables las empresas transnacionales (cuya capacidad de absorber riesgos es mayor que la de las nacionales) tendrán una mayor participación en la inversión privada total. Ambas predicciones son empíricamente falseables.

\footnotetext{
2 Se podría suponer que después de un alto grado de equidad, la curva se hace descendente. Un exceso de igualdad puede generar desincentivos para los inversionistas. La curva tendría, entonces, la forma de una $\mathrm{U}$ invertida.
}

\footnotetext{
3 Siguiendo a Krugman, denominaremos ventajas competitivas a las que afectan al comercio intraindustrial.
} 


\section{IV}

\section{Información empírica}

Un nuevo conjunto de datos sobre equidad presentado recientemente por el Banco Mundial (Deininget y Squire, 1996) se basa en una muestra de 108 países que abarca desde los años cincuenta hasta los noventa, con un total de 682 observaciones. En esta muestra, América Latina (con 20 países y 100 observaciones) aparece como la región que exhibe más desigualdad en el mundo (cuadro 1). Su coeficiente de Gini tiene una media de 0.50 , mientras que el de los países capitalistas avanzados es de 0.33 , y el de los "tigres" asiáticos alrededor de 0.35 . Este orden se mantiene si se utiliza como índice la razón entre la participación en el ingreso de uno y otro de los quintiles extremos.

En realidad, hay otros dos resultados interesantes que emergen de los datos de Deininger y Squire (1996, cuadro 1); primero, el orden de desigualdad entre las regiones se mantiene a través del tiempo; y segundo, los cambios en los coeficientes de Gini no son radicales dentro de cada región. ${ }^{4}$ Estos datos son consistentes con nuestra hipótesis de la persistencia de la desigualdad pronunciada, cuando ésta es una condición inicial de la economía. ${ }^{5}$
América Latina ha perdido participación en el flujo de inversiones extranjeras directas. Desde el primer quinquenio de los años ochenta esa participación ha estado bajando, sea que se la mida con respecto a todos los países o sólo con respecto a los países en desarrollo (cuadro 2). Perú, uno de los países de la región donde la desigualdad económica es más pronunciada, tuvo en 1988-1992 una marcada inestabilidad

CUADRO 2

América Latina: Inversiones extranjeras directas (Promedios anuales)

\begin{tabular}{lrrr} 
& \multicolumn{3}{c}{ Miles de millones de dólares } \\
\cline { 2 - 3 } & 1981-1985 & 1986-1990 & 1991-1993 \\
\hline A. Todos los países & 50 & 155 & 171 \\
B, Países en desarrollo & 13 & 25 & 57 \\
C. América Latina y el Caribe & 6 & 8 & 17 \\
C/B (\%) & 46.2 & 32.0 & 30.0 \\
C/A $(\%)$ & 12.0 & 5.2 & 9.9 \\
\hline
\end{tabular}

Fuente: CEPAL, 1995, cuadro II. 1, p. 55.

\begin{tabular}{lccc}
\hline & $\begin{array}{c}\mathrm{N}^{\circ} \mathrm{de} \\
\text { observaciones } \\
\text { (países por año) }\end{array}$ & $\begin{array}{c}\text { Coeficiente } \\
\text { de Gini }\end{array}$ & $\begin{array}{c}\text { Razón entre la participación } \\
\text { en el ingreso de uno } y \text { otro } \\
\text { de los quintiles extremos }\end{array}$ \\
\hline Ámérica Latina & 100 & 0.50 & 16.02 \\
África subsahariana & 40 & 0.45 & 11.61 \\
Medio oriente y Noráfrica & 20 & 0.41 & 7.14 \\
Este asiático y el Pacífico & 123 & 0.36 & 5.15 \\
Asia del sur & 60 & 0.34 & 6.63 \\
Países industrializados & 238 & 0.26 & 4.05 \\
Europa oriental & 101 & 0.36 & 7.80 \\
Total & 682 & & 0.36 \\
\hline
\end{tabular}

Fuente: Deininger y Squire, 1996, cuadro 1.

\footnotetext{
${ }^{4}$ Aun a nivel de país, hay una notable estabilidad en el coeficiente de Gini: para los países con más de diez observaciones, el coeficiente de variabilidad de Pearson no sobrepasa el $12 \%$. Este dato empírico plantea cuestiones interesantes: ¿es la equidad una caracteristica estructural de cada sociedad, una condición inicial difícil de modificar sustantivamente?
}

\footnotetext{
${ }^{5}$ El sabio Alexander Humboldt expresó en sus escritos que una de las características que más le impresionó en sus visitas a América era la tremenda desigualdad económica y social. Ciento cincuenta años después seguimos hablando del mismo tema como problema central de América Latina.
} 
política. En ese período la inversión extranjera ingresó al país en pequeñas magnitudes y empezó a aumentar en 1993 (cuadro 3). Estos datos son coherentes con nuestra hipótesis de que las inversiones se dirigen en mayor proporción a sociedades más igualitarias.

La relación entre equidad e inversión, que es una ecuación estructural en nuestro sistema teórico - ecuación (8) - , es puesta a prueba estadística en un estudio de Alesina y Perotti (1993). Para una muestra de 70 países, con datos del período 1960-1985, ellos encuentran una correlación negativa entre la equidad y la inestabilidad sociopolítica, por un lado, y entre la inestabilidad sociopolítica y la inversión, por otro. En esta muestra, los 16 países latinoamericanos incluidos exhibieron los mayores grados de desigualdad y de inestabilidad sociopolî́tica, así como las menores tasas de inversión (Ibid., cuadros 4 y 5, y p. 19).

La interpretación que hacen Alesina y Perotti de sus resultados, así como las conclusiones de política que derivan de ellos, tienen sin embargo una dificultad lógica. En su modelo estos autores toman la distribución del ingreso como una variable explicativa, es decir, como una variable exógena en el proceso económico. No hay teoría económica que avale tal propuesta. La manera de resolver esta dificultad lógica sería considerar un sistema teórico como el desarrollado aquí, en el que la inversión del período presente depende del grado de inestabilidad sociopolítica del mismo período, y donde esta última variable depende del grado de equidad del período anterior. En este sistema dinámico, y a falta de datos sobre la distribución de los acervos, la distribución del ingreso puede ser utilizada como una condición inicial en la trayectoria temporal de las variables endógenas.

En realidad, en el análisis estadístico que los autores indicados llevan a cabo, ellos utilizan la distribución del ingreso del inicio del período de análisis (1960) y para las demás variables, las medias del período 1960-1985. Pero entonces la interpretación de los resultados no puede ser la que ellos hacen, que la inversión depende de la desigualdad: "la desigualdad del ingreso aumenta la inestabilidad política, la que a su vez disminuye la inversión" (Alesina y Perotti, 1993, p. 18). Ambas son variables endógenas. Si esos datos se consideran generados por un sistema dinámico, la relación de causalidad tendría que ser otra: la alta concentración del ingreso, como condición inicial, aumenta el riesgo de inestabilidad sociopolítica, lo cual lleva a una trayectoria con menores inversión y crecimiento.

Además, la conclusión de política de Alesina y Perotti tendría que ser reformulada, Ellos concluyen que la redistribución del ingreso tiene efectos netos impredecibles pues la mayor presión tributaria, necesaria para la redistribución, reduciría los incentivos para la inversión. Pero este efecto puede ser de nivel y no de tasa. Esta confusión es similar a la que existe cuando se considera que las barreras al comercio internacional basadas en aranceles de protección, que son también tasas impositivas, constituyen un factor que afecta el crecimiento económico. Como bien señala Lucas (1988 pp. 12 y 13), la liberalización de esas ba-

CUADRO 3

Flujos de inversión extranjera directa a Perú y a un grupo de países latinoamericanos ${ }^{81}$ 1988-1993

(Millones de dólares)

\begin{tabular}{|c|c|c|c|c|c|c|}
\hline & 1988 & 1989 & 1990 & 1991 & 1992 & 1993 \\
\hline Perú & $20 ́$ & 59 & 41 & -7 & 127 & 349 \\
\hline Flujos normales & 26 & 59 & 41 & -7 & 43 & 60 \\
\hline Conversión de deuda & - & - & - & - & - & - \\
\hline Privatizaciones & - & - & - & - & 140 & 289 \\
\hline Grupo & 7961 & 7469 & 6951 & 11062 & 12271 & 44420 \\
\hline Flujos normales & 3613 & 4570 & 3894 & 7512 & 9826 & 8388 \\
\hline Conversión de deuda & 4154 & 2784 & 1841 & 305 & 133 & 25 \\
\hline Privatizaciones & 194 & 115 & 1216 & 3245 & 2312 & 6007 \\
\hline Perú/grupo \{\%\} & 0.3 & 0.8 & 0.6 & $-0,1$ & 1.0 & 2.42 \\
\hline
\end{tabular}

Fuente: CEPAL, 1995, cuadro IX. 4, p. 206.

a Incluye Argentina, Brasil, Chile, Colombia, México, Perú y Venezuela. 
rreras podría tener un efecto sobre el nivel pero no sobre la tasa de crecimiento. Si adoptamos la teoría de Lucas de que la distribución del ingreso afectana la tasa de crecimiento, la conclusión lógica del estudio de Alesina y Perotti, a la luz de nuestra reinterpretación de sus resultados empíricos, tendría que ser otra: redistribuir ingresos a modo de cambio en las condiciones iniciales llevaría a una trayectoria distinta, con mayor inversión y crecimiento; es decir, tendría un efecto sobre la tasa de crecimiento.

A juzgar por su participación en el comercio mundial, América Latina ha perdido competitividad desde los años sesenta. El cuadro 4 muestra esta tendencia con claridad. Entre 1960 y 1992 la región redujo esa participación casi a la mitad, de $7.7 \%$ a $3.7 \%$. Los países desarrollados concentran la mayor parte del comercio mundial y han elevado aun más su participación. Pero el grupo que más ha ganado en competitividad es el de los llamados "tigres" asiáticos (República de Corea, la provincia china de Taiwán, Singapur, Hong Kong, Indonesia, Malasia y Tailandia).

\begin{tabular}{|c|c|c|c|c|}
\hline & \multicolumn{4}{|c|}{$\begin{array}{l}\text { Participación de América Latina en } \\
\text { el comercio mundial } \\
\text { (Porcentajes) }\end{array}$} \\
\hline & $\begin{array}{l}\text { América } \\
\text { Latina }\end{array}$ & $\begin{array}{c}\text { Países } \\
\text { desarrollados }\end{array}$ & $\begin{array}{c}\text { "Tigres" } \\
\text { asiáticos }\end{array}$ & Otros \\
\hline 1960 & 7.7 & 65.9 & 3.4 & 23.0 \\
\hline 1970 & 5.5 & 70.9 & 3.0 & 20.6 \\
\hline 1980 & 5.5 & 62.6 & 6.0 & 25.9 \\
\hline 1990 & 3.9 & 71.4 & 10,1 & 14.6 \\
\hline 1992 & 3.7 & 71.5 & 11.5 & 13.3 \\
\hline
\end{tabular}

Fuente: CEPAL, 1995, cuadro 1. 4, p. 35.

a República de Corea, provincia china de Taiwán, Singapur, Hong Kong, Indonesia, Malasia y Tailandia.

Una muestra de ocho países de América Latina nos indica el mismo patrón regional: pérdida de participación en el mercado mundial, aunque con diferencias de grado que bien vale la pena señalar (cuadro 5). La pérdida de participación ha sido más severa en los

CUADRO 5

América Latina (ocho países): Participación relativa en el mercado mundial, 1964-1993

(Porcentajes)

\begin{tabular}{|c|c|c|c|c|c|c|c|c|}
\hline & Argentina & Brasil & Chile & Colombia & Costa Rica & México & Paraguay & Perú \\
\hline 1964 & 0.90 & 0.91 & 0.38 & 0.35 & 0.07 & 0.67 & 0.03 & 0.42 \\
\hline 1965 & 0.87 & 0.93 & 0.37 & 0.32 & 0.07 & 0.67 & 0.03 & 0.39 \\
\hline 1966 & 0.85 & 0.93 & 0.44 & 0.27 & 0.07 & 0.64 & 0.03 & 0.41 \\
\hline 1967 & 0.74 & 0.84 & 0.43 & 0.26 & 0.07 & 0.58 & 0,02 & 0.38 \\
\hline 1968 & 0.62 & 0.86 & 0.39 & 0.25 & 0.08 & 0.57 & 0,02 & 0.39 \\
\hline 1969 & 0.64 & 0.92 & 0.43 & 0.24 & 0.08 & 0.57 & 0.02 & 0.34 \\
\hline 1970 & 0.61 & 0.94 & 0.43 & 0.25 & 0.08 & 0.48 & 0.02 & 0.36 \\
\hline 1971 & 0.53 & 0.89 & 0.30 & 0.21 & 0.07 & 0,46 & 0.02 & 0.27 \\
\hline 1972 & 0.50 & 1.03 & 0.22 & 0.21 & 0.07 & 0.44 & 0.02 & 0.24 \\
\hline 1973 & 0.61 & 1.15 & 0.23 & 0.22 & 0.06 & 0.42 & 0.02 & 0.21 \\
\hline 1974 & 0.50 & 1.00 & 0.31 & 0.19 & 0.06 & 0,37 & 0.02 & 0.19 \\
\hline 1975 & 0.36 & 1.06 & 0.19 & 0.18 & 0.06 & 0.36 & 0.02 & 0.16 \\
\hline 1976 & 0.43 & 1.10 & 0.23 & 0.20 & 0.06 & 0.37 & 0.02 & 0.15 \\
\hline 1977 & 0.54 & 1.16 & 0.21 & 0.23 & 0.08 & 0.40 & 0.03 & 0.17 \\
\hline 1978 & 0.52 & 1.05 & 0.20 & 0.25 & 0.07 & 0.50 & 0.02 & 0.16 \\
\hline 1979 & 0.51 & 0.99 & 0.25 & 0.22 & 0.06 & 0.58 & 0.02 & 0.23 \\
\hline 1980 & 0.42 & 1.61 & 0.25 & 0.21 & 0.05 & 0.82 & 0.02 & 0.21 \\
\hline 1981 & 0.49 & 1.24 & 0.20 & 0.16 & 0.05 & 1.05 & 0.02 & 0.17 \\
\hline 1982 & 0.44 & 1.16 & 0.21 & 0.17 & 0.05 & 1.22 & 0.02 & 0.19 \\
\hline 1983 & 0.46 & 1.29 & 0.23 & 0.18 & 0.05 & 1.29 & 0.02 & 0.18 \\
\hline 1984 & 0.45 & 1.50 & 0.20 & 0.19 & 0.06 & 1.36 & 0.02 & 0.17 \\
\hline 1985 & 0.46 & 1.41 & 0.21 & 0.20 & 0.05 & 1.22 & 0.02 & 0.16 \\
\hline 1986 & 0.34 & 1.19 & 0.21 & 0.26 & 0.06 & 0.82 & 0.01 & 0.13 \\
\hline 1987 & 0.27 & 1.11 & 0.22 & 0.20 & 0.05 & 0.89 & 0.02 & 0.11 \\
\hline 1988 & 0.34 & 1.25 & 0.26 & 0.19 & 0.05 & 0.77 & 0.02 & 0.10 \\
\hline 1989 & 0.33 & I. .17 & 0.28 & 0.20 & 0.05 & 0.79 & 0.03 & 0.12 \\
\hline 1990 & 0.37 & 0.93 & 0.25 & 0.20 & 0.04 & 0.81 & 0,03 & 0.10 \\
\hline 1991 & 0.35 & 0.92 & 0.26 & 0.21 & 0.05 & 0,79 & 0.02 & 0.10 \\
\hline 1992 & 0.33 & 0.98 & 0.27 & 0.19 & 0.05 & 0.75 & 0.02 & 0.09 \\
\hline 1993 & ... & 1.05 & 0.25 & , & 0.06 & 0.82 & $\ldots$ & - \\
\hline
\end{tabular}

Fuente: FMI, 1974 y 1994. 
casos de Argentina y Perú, menos severo en Colombia y Costa Rica. Chile perdió participación entre 1964 y 1986, para luego empezar a recuperarla, pero sin poder alcanzar en el período su nivel de inicios de los años sesenta. Paraguay ha mantenido casi el mismo nivel de participación aunque con grandes oscilaciones. Brasil exhibió éxitos en el período 1964-1984, pero desde entonces ha perdido posiciones hasta lle- gar a niveles similares al de inicios de los años sesenta. El país relativamente más exitoso de la muestra es México. En 1964-1976 perdió posiciones, en 1977 1985 las ganó de manera sustancial, para perderlas en parte en 1986-1989 y estabilizarse desde entonces en un nivel superior al de inicios de los años sesenta. En suma, no tenemos en esta muestra latinoamericana un caso comparable al de los "tigres" asiáticos.

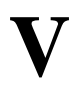

\section{El papel de la dotación de recursos naturales}

La importancia de la equidad en la determinación de la inversión privada depende, como dijimos antes, del grado de integración del sector exportador a la economía nacional. La inversión privada buscaría explotar las ventajas absolutas, relativas o competitivas según fuesen la condiciones iniciales de un país en cuanto a dotación de recursos y equidad. Una sociedad con una amplia dotación de recursos naturales solamente y un alto grado de desigualdad inicial sólo podría dar lugar a inversiones que exploten sus ventajas absolutas y algo de sus ventajas comparativas, Pero con tales inversiones al país le sería difícil salir del atraso social.

Una dotación significativa de recursos naturales puede resultar contraproducente. Al país que la tenga puede serle difícil desarrollarse sobre la base de ventajas absolutas. La explotación de las ventajas absolutas de un país no requiere que esté tenga estabilidad sociopolítica. La actividad exportadora puede operar en un enclave. Cuanto más integrado esté el sector exportador al sistema productivo del país, más importante será el orden social, y por la tanto la equidad, para atraer inversiones privadas que desarrollen los sectores de exportación.

En consecuencia, sociedades bien dotadas con recursos naturales tendrán un nivel de inversión extranjera mayor, dado el grado de desigualdad en la sociedad. Esta es otra predicción empírica del modelo.

¿En qué bienes se ha especializado América Latina? En casi toda su historia, y hasta fines de los años setenta, la región se ha especializado principalmente en productos primarios, como los mineros, petroleros, pesqueros y agrícolas; en las últimas décadas se han desarrollado las exportaciones de manufacturas (cuadro 6).

Con los productos primarios, la región exporta su abundante dotación de recursos naturales, incluidos los

\begin{tabular}{lrrrrr} 
CUADRO 6 & $\begin{array}{l}\text { América Latina y el Caribe: } \\
\text { Estructura de las exportaciones } \\
\text { (Porcentajes) }\end{array}$ \\
& 1962 & 1970 & 1980 & 1990 & 1992 \\
\hline & 52.4 & 47.1 & 31.2 & 28.3 & 29.8 \\
\hline Producios agrícolas & 13.1 & 18.2 & 10.4 & 11.7 & 9.7 \\
Metales y minerales & 29.1 & 22.9 & 40.6 & 26.5 & 21.6 \\
Combustibles & 5.2 & 11.5 & 17.3 & 32.9 & 38.5 \\
Manufacturas & 0.2 & 0.3 & 0.5 & $\mathbf{0 . 6}$ & 0.4 \\
Otros & 100.0 & 100.0 & 100.0 & 100.0 & 100.0 \\
Total & & & & & \\
\hline
\end{tabular}

Fuente: CEPAL, 1995, cuadro 1.6, p. 39.

climáticos. Sus depósitos de minerales y petróleo le permiten exportar estos productos. Por su biodiversidad, genera productos pesqueros, coca, café, algodón, azúcar, espárragos, lana de auquénidos, es decir, bienes que no se pueden producir en cualquier parte del mundo, ni en cualquier época. El turismo es también la exportación de un recurso natural, que se basa en el clima o que toma la forma de un recurso histórico.

Una combinación de ventajas absolutas y comparativas está en la base de esta especialización. Pero, salvo respecto de algunos productos agrícolas, la explotación de estos recursos naturales no ha significado que la región exporte bienes que hagan uso intensivo de mano de obra, su factor relativamente más abundante. Los minerales, metales y combustibles, en cambio, hacen uso relativamente más intensivo de capital. Con ellos, América Latina exporta bienes cuya rentabilidad económica es alta. Así, una parte importante de sus exportaciones no depende de las variaciones del precio internacional, ni del tipo de cambio, ni 
del salario, al menos en grado significativo, sino de la inversión.

La producción agrícola, que hace uso relativamente intensivo de mano de obra, especialmente de la no calificada, ha perdido importancia relativa en las exportaciones (cuadro 6). En términos absolutos, los datos indican que el volumen de exportaciones agrícolas creció $4 \%$ anualmente en los años setenta, pero esa tasa bajó a $2.3 \%$ en los ochenta (CEPAL, 1995, p. 70).

Aunque es cierto que la expansión de las exportaciones de manufacturas se ha dado en toda la región, los datos del cuadro 6 esconden diferencias marcadas entre los países. Cerca del $75 \%$ de las exportaciones de manufacturas de 1993 corresponde sólo a Brasil y México (CEPAL, 1995, cuadro III.5, p. 77). Sin estos países, la media simple para la región es de $18 \%$, en lugar del 39\% que aparece en el cuadro.

Dentro de la actividad manufacturera se puede distinguir un sector de bienes tradicionales que hace uso relativamente intensivo de mano de obra: el de los textiles, confecciones y calzado. En él la abundancia relativa de la mano de obra parece desempeñar un papel importante en la competitividad. La ubicación de la maquila de estos productos, desarrollada por las empresas transnacionales en varios países de la región, se basaría precisamente en la existencia de mano de obra barata.

No es posible contar con datos para poner a prueba la hipótesis de que las inversiones en sectores primarios requieren menos estabilidad sociopolítica, pero hay una historia que sirve para ilustrar esa hipótesis. Cusiana, en Colombia, es el yacimiento petrolero más grande de América Latina de los últimos 20 años. La inversión en él es de 6000 millones de dólares. La empresa petrolera, que era extranjera, tuvo que interrumpir sus trabajos de exploración en varias ocasiones porque la guerrilla invadió y destruyó sus campos; para rechazar a la guerrilla se han destacado soldados en Cusiana (El Comercio, 1996", p. E6). Este yacimiento se pondrá en explotación de inmediato a pesar de que Colombia es un país plagado de pobreza, caos político, guerrilla y narcotráfico. Parece que no hay requisitos previos en términos de estabilidad sociopoiítica para explotar petróleo; su producción es de enclave, físico y económico.

La otra hipótesis es que distintas dotaciones de factores dan lugar a diferentes patrones de comercio y a distintos grados de equidad distributiva. Más específicamente, la inversión que desarrolla un sector exportador basado en la explotación de recursos naturales llevaría a una mayor concentración del ingreso. Esta hipótesis fue puesta a prueba empírica por Bourguignon y Morrisson (1989, cap. II) en un análisis de corte transversal para 1970 . La muestra incluía 20 países en desarrollo, de los cuales seis eran de América Latina (Argentina, Chile, Colombia, Costa Rica, Perú y Uruguay).

Para el análisis estadístico, el estudio de Bourguignon y Morrisson incluyó como variable endógena la distribución del ingreso (medido por la participación de algunos deciles en el ingreso nacional); como variables exógenas, el grado de protección comercial, el peso en el producto interno bruto de la exportación agrícola y de las exportaciones de productos mineros y petroleros, la estructura de la propiedad agrícola y la educación. Sus resultados muestran que la protección tiene un efecto negativo sobre la equidad; también la especialización basada en recursos naturales, salvo cuando la exportación agrícola proviene principalmente de pequeños predios, Los patrones de comercio basados en las ventajas absolutas son concentradores de ingresos y los basados en las ventajas comparativas de la agricultura dependen del grado de concentración de la propiedad de la tierra.

Tales resultados se pueden reinterpretar a la luz de nuestra teoría: las sociedades inequitativas y bien dotadas con recursos naturales atraerán inversiones dirigidas a explotar principalmente sus recursos naturales, con lo cual continuarán siendo inequitativas e inestables. Con este mecanismo se mantiene su condición inicial de sociedades muy desiguales.

En esta perspectiva teórica, el gasto público social puede ser visto como un instrumento para poner pisos a los ingresos y el bienestar de la población, y así darle estabilidad al sistema social y político. A estas acciones se las puede denominar "política social". Pero ese piso tendría que ser establecido como un conjunto de derechos: una redistribución para financiar derechos económicos. Esto significa que los bienes y servicios involucrados tendrían que ser retirados del juego del mercado y del juego político-electoral. La estabilidad social, al igual que la democracia, es un bien público: una vez establecida, nadie puede ser excluido de su consumo. Es evidente, entonces, que el gasto público social es una inversión en un bien público: la estabilidad social.

Sin embargo, la experiencia latinoamericana no va en esa dirección. Un estudio de la CEPAL (1994) muestra que en los años ochenta e inicios de los noventa el gasto social en un grupo de países de la región no representaba una proporción significativa de! PIB, que sus variaciones no eran anticíclicas a las del PIB, y que no tenía efectos significativamente progresivos en la 
distribución. Claramente, el sistema político imperante no ha utilizado la política social para modificar la situación de desigualdad pronunciada en que se encuen-

\section{VI}

\section{Conclusiones}

La pregunta que ha guiado el presente estudio es si una sociedad puede llegar a ser desarrollada partiendo de un alto grado de desigualdad. En particular, si un país puede ser competitivo en la economía internacional cualquiera sea su grado de desigualdad actual.

Si las condiciones iniciales de una economía se caracterizan por una dotación de recursos naturales y un alto grado de desigualdad, la inversión privada se dirigirá a explotar esos recursos y a producirlos en enclaves. Con estas condiciones iniciales será difícil que la inversión desarrolle ventajas comparativas y competitivas. Así, el comercio internacional no contribuirá a reducir el exceso de mano de obra que pueda tener esta economía, ni la desigualdad se reducirá con el comercio.

$\mathrm{Si}$ alguna relación pudiera existir entre comercio y equidad, sería más bien en el sentido contrario: para desarrollar las ventajas comparativas y competitivas es preciso que el país exhiba una desigualdad poco pronunciada. La lógica de los inversionistas los llevaría a colocar sus inversiones en países con estabilidad sociopolítica, la cual dependería del grado de equidad de la sociedad. No es que las exportaciones induzcan el crecimiento económico, como se dice usualmente; las exportaciones son endógenas. Es la inversión la que genera crecimiento y mayor productividad y por esta vía el país gana competitividad. Y cuanto más intensivos en orden social sean los bienes que exporte un país, mayor será el efecto de la equidad sobre sus exportaciones netas. La competitividad internacional de un país depende de su grado de equidad: esta es la teoría que se ha desarrollado en el presente trabajo.

Puesta a prueba empírica, la teoría muestra un grado aceptable de consistencia con los datos de América Latina. Una predicción de esta teoría es que el desempeño económico de los países depende del grado de equidad y de la dotación de factores con que ellos partieron. A inicios de los años sesenta, America Latina partió con una desigualdad pronunciada y con una dotación abundante de recursos naturales y mano de obra no calificada; la República de Corea y la provin- tra la mayoría de los países de América Latina. Este resultado empírico también es consistente con la teoría propuesta aquí. cia china de Taiwán partieron con condiciones opuestas: mayor equidad y dotación de capital humano. Las trayectorias de crecimiento, equidad y competitividad de nuestra región y de ambas sociedades asiáticas han sido como predice la teoría: hoy día hablamos de "tigres" asiáticos y no de "tigres" latinoamericanos.

Que el capital de largo plazo fluye en mayor proporción hacia los países más igualitarios es otra predicción de la teoría que es coherente con las tendencias de la economía mundial. En el período actual hay una mayor integración económica mundial, especialmente financiera. Se han abandonado los controles sobre las tasas de cambio y los mercados de capitales se han globalizado. Según un estudio de la Organización de Cooperación y Desarrollo Económicos (OCDE), los flujos mundiales de inversión extranjera directa aumentaron en las últimas décadas a tasas sin precedentes, tres a cinco veces mayores que la tasa de crecimiento del flujo de comercio internacional (Ornan, 1996, p. 26). Esto indica que los países (incluidos los del tercer mundo) no pueden ser mirados como distintos en su dotación de capital, ya que el capital se puede considerar internacionalmente móvil.

En suma, las productividades relativas de los países (lo que hemos definido aquí como competitividad de largo plazo) dependerían de la distribución de las inversiones entre ellos; estas inversiones dependerían de la inversión pública y del grado de estabilidad sociopolítica de los países receptores; y este grado dependería a su vez de la equidad. Luego, la competitividad dependería de la equidad. Debido a que la estabilidad sociopolítica necesitaría un grado de organización de la sociedad para mantener una equidad tolerable, es evidente que la competitividad́ no es sólo una cuestión de eficiencia microeconómica o sectorial; tampoco es sólo una cuestión de balances macroeconómicos; es también una cuestión social. Las sociedades compiten unas con otras para atraer las inversiones privadas con el fin de hacerse competitivas; pero compiten, entre otros factores, con su grado de equidad. 


\section{Bibliografía}

Alesina, A. y R. Perotti (1993): Income Distribution, Polítical Instability, and Investment, Cambridge, Massachusetts, National Bureau of Economic Research, Paper 4486, octubre.

Barro, R. (1990): Macroeconomics. Nueva York, Wiley \& Sons Inc, Bourguignon, F. y C. Morrisson (1989): External Trade and Income Distribution, París, Organización de Cooperación y Desarrollo Económicos (OCDE).

CEPAL (Comisión Económica para América Latina y el Caribe) (1990): Transformación productiva con equidad, LC/G.1601-P, Santiago de Chile. Publicación de las Naciones Unidas, $\mathrm{N}^{\circ}$ de venta S.90.II.G.6.

(1994): El gusto social en América Latina: Un examen cuantitativo y cualitativo. Cuadernos de la CEPAL, $\mathrm{N}^{\circ} 73$, LC/G.1854-P, Santiago de Chile, Publicación de las Naciones Unidas, $\mathrm{N}^{\circ}$ de venta S.95.H.G.9.

\{1995): América latina y el Caribe: políticas para mejorar la inserción en la economía mundial, LC/G,1800/Rev.1-P, Santiago de Chile, Publicación de las Naciones Unidas, $\mathrm{N}^{\mathrm{c}}$ de venta S.95.II.G.6.
Deininger, K. y L. Squire (1996): A new data set measuring income inequality, The World Bank Economic Review, vol. 10, $\mathrm{N}^{\circ} 3$, Washington, D.C., Banco Mundial.

El Comercio (1996): Lima, 3 de enera.

Figueroa, A. (1993): Crisis distributiva en el Perú, Lima, Pontificia Universidad Católica del Perú, Fondo Editorial.

FMI (Fondo Monetario Internacional) (1974): Estadísticas financieras internacionales. Anuario 1994, Washington D.C.

(1994); Estadísticas financieras internacionales. Anuario 1994, Washington D.C.

Hicks, J. (1989): A Market Theory of Money, Oxford, Reino Unido, Clarendon Press,

Krugman, P. (1995): The Age of Diminished Expectations, Cambridge, Massachusetts, The MIT Press.

Lucas, R. (1988): On the mechanics of economic development, Journal of Monetary Economías, vol. 22, $\mathrm{N}^{\circ}$ 1, Amsterdam, Países Bajos, North-Holland Publishing Company, julio.

Ornan, C, (1996): Desafíos políticos de la globalización y regionulización, Lima, Fundación Friedrich Ebert.

Solow, R. (1990): The Labor Market as a Social Institution, Oxford, Reino Unido, Basil Blackwell. 